\title{
Pseudo-rearrangement of the MLL gene at chromosome 11q23: a cautionary note on genotype analysis of leukaemia patients
}

\author{
M Stanulla, H J Schünemann, S Thandla, M L Brecher, P D Aplan
}

\begin{abstract}
Aims-The MLL gene on chromosome 11 q23 is frequently disrupted by chromosomal translocations in association with haematological malignancies. Recently, a specific site within the $8.3 \mathrm{~kb}$ MLL breakpoint cluster region that is cleaved during the early stages of apoptosis has been identified. Because MLL gene rearrangements are used to identify patients with high risk leukaemia, it was the aim of this study to determine whether this DNA cleavage event could be triggered in diagnostic bone marrow samples solely through ex vivo incubation at room temperature.

Methods-Pretreatment bone marrow samples were collected from six paediatric leukaemia patients. Genomic DNA for Southern blot analysis of MLL gene rearrangements was isolated immediately after samples were obtained and compared to genomic DNA isolated after incubation of specimens for 24-60 hours at room temperature, simulating delays in processing that might occur when samples are delivered to reference laboratories. In addition, cryopreserved samples from 70 paediatric leukaemia patients were screened for evidence of site specific MLL cleavage.
\end{abstract}

Department of Molecular Medicine, Roswell Park Cancer Institute, Buffalo, NY 14263 , USA

M Stanulla

Department of Pediatrics, Roswell Park Cancer Institute, Buffalo, NY 14263, USA

S Thandla

M L Brecher

P D Aplan

Department of Medicine, School of Biomedical Sciences, State University of

New York at Buffalo, Buffalo, NY 14214, USA

H J Schünemann

Correspondence to: Dr Aplan.

email: paplan@sc3101.med. buffalo.edu

Accepted for publication 20 January 1998 leukaemia (ALL) and acute myeloid leukae (AML), especially in infants less than 1 year of age. $^{5-7}$ Furthermore, many patients with therapy related acute myeloid leukaemia associated with chemotherapeutic agents targeting topoisomerase II display MLL translocations. ${ }^{89}$ At least 30 different MLL translocation partners have been identified, leading to the designation of MLL as a "promiscuous" oncogene..$^{610}$ MLL aberrations have repeatedly been shown to confer a poor prognosis in ALL and certain types of AML, suggesting aggressive disease that is less susceptible to conventional therapeutic regimens, and potential benefits from more intensive therapy. ${ }^{5-7}$ Indeed, in some recent treatment schemes, a rearranged MLL gene leads to patient stratification into high risk groups with treatment approaches including allogenic bone marrow transplantation. ${ }^{6711}$ Therefore, careful assessment of a leukaemia patient's MLL status is of considerable clinical importance.

Conventional cytogenetic analysis may not detect MLL aberrations for a variety of reasons including karyotype misinterpretation, lack of adequate mitosis, and submicroscopic MLL gene rearrangements. The recent observation that a partial tandem duplication of the MLL gene might occur in as many as $10 \%$ of adult AML patients with normal cytogenetic analyses ${ }^{12}$ underscores the need for additional methods for identifying MLL gene rearrangements. Two complementary molecular approaches have been used to detect MLL gene rearrangements not identified by conventional cytogenetic analysis. ${ }^{67}$ Multiplex reverse transcription-polymerase chain reaction (RTPCR) has been used to screen samples for fusion transcripts produced by the most common MLL translocations, such as the $\mathrm{t}(4 ; 11), \mathrm{t}(9 ; 11)$, and $\mathrm{t}(11 ; 19)$ translocations. $^{6}$ This approach is limited to those MLL translocations where the fusion partner is known and characterised, and is further limited by the need for intact mRNA. A second approach takes advantage of the observation that almost all MLL gene rearrangements occur within a limited $8.3 \mathrm{~kb}$ breakpoint cluster region, and can be detected by Southern blot hybridisation using an MLL cDNA probe. ${ }^{5}$ In this analysis, identification of a nongermline sized MLL fragment is presumed to represent a breakpoint within the MLL breakpoint cluster region generated by a chromosomal translocation, and is referred to as an MLL gene rearrangement. Because of the poor clinical outcome of infants with MLL gene rearrangements, ${ }^{13-16}$ some recent classification schemes assign infants with non-informative cytogenetics to a high risk group if they 
Table 1 Clinical characteristics of leukemia patients demonstrating pseudo-rearrangements

\begin{tabular}{|c|c|c|c|c|c|c|}
\hline Patient & Age & Sex & Diagnosis & $\begin{array}{l}W B C \\
\left(\times 10^{9} / L\right)\end{array}$ & $\begin{array}{l}\text { Blasts in bone } \\
\text { marrow (\%) }\end{array}$ & Karyotype \\
\hline RUPN 96 & 10 years & Male & B-precursor ALL & 4.2 & 72 & $46 ; \mathrm{XY}$ \\
\hline RUPN 97 & 15 months & Male & B-precursor ALL & 11.7 & 91 & $\begin{array}{l}58 ; \mathrm{XY} \\
+4,+6,+9,+10,+14,+18,+18 \\
+21,+21,+\operatorname{mar} 1,+\operatorname{mar} 2\end{array}$ \\
\hline RUPN 42 & 9 years & Female & B-ALL (L3) & 13.0 & 92 & Inadequate mitosis \\
\hline
\end{tabular}

RUPN refers to unique patient number; RUPN 96 was a relapsed patient; L3, phenotype as classified by the

French-American-British (FAB) scheme.

ALL, acute lymphoblasic leukaemia; WBC, peripheral white blood cell count at diagnosis (or relapse for RUPN 96).

demonstrate MLL gene rearrangements by Southern blot analysis. ${ }^{6}{ }^{11}$

A unique site within the MLL breakpoint cluster region that is highly sensitive to DNA double stranded breaks induced by treatment of intact cells with topoisomerase II inhibitors (such as etoposide and doxorubicin) has been identified. ${ }^{17}$ This site specific cleavage generates additional non-germline sized fragments on Southern blot hybridisation using the MLL cDNA probe commonly used to genotype the MLL gene. ${ }^{17}$ Subsequent studies demonstrated that induction of the same site specific cleavage event within the MLL breakpoint cluster region could also be caused by various other stimuli, and could be attributed to the higher order chromatin fragmentation that occurs during the early stages of apoptosis. ${ }^{18}$ Apoptosis, or programmed cell death, is a genetically conserved programme that is triggered by a wide variety of stimuli (chemotherapeutic agents, tumour necrosis factor $\alpha$, and serum deprivation) and can be described as a cell's active commitment to suicide, involving distinct cytoplasmic and nuclear events. ${ }^{19} 20$

Evaluation of a patient's MLL gene status often requires bone marrow samples to be processed at reference laboratories, a procedure that can easily lead to a 48 hour (or more) delay in processing of the sample, especially if samples are shipped over a weekend. Because we have demonstrated recently that serum deprivation can lead to apoptosis and site specific MLL cleavage of leukaemia cell lines ${ }^{18}$ we wondered whether an analogous phenomenon could be observed in primary leukaemic samples. In this report, we show that apoptotic higher order chromatin fragmentation can occur in heparinised bone marrow specimens in a time dependent manner, generating a pseudo-rearrangement of the MLL gene in

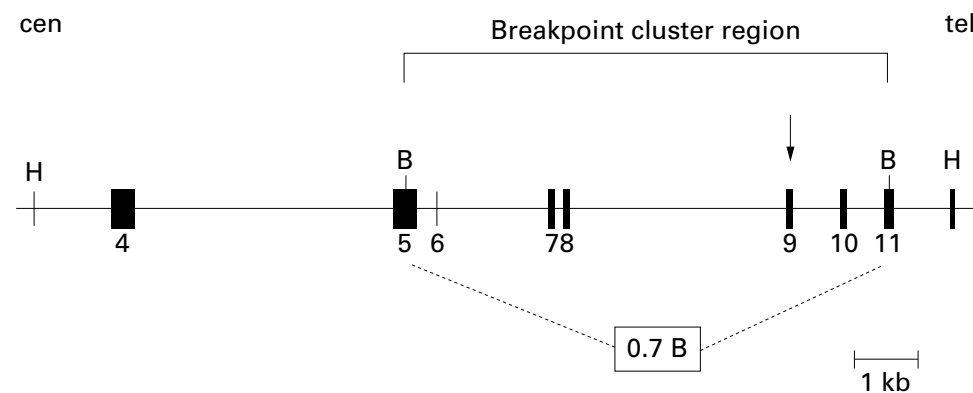

Figure 1 Pseudo-rearrangements of the MLL gene in leukaemia patients. Partial restriction map of the $M L L$ gene. The breakpoint cluster region is bracketed. Exons are shown as solid boxes. Chromosomal orientation from centromere (cen) to telomere (tel) is as indicated. The point of site specific cleavage during apoptosis is indicated by a downward arrow. The region covered by the $0.7 B M L L c D N A$ probe is indicated. Restriction enzyme sites are: B, BamHI; and H, Hind III.
Southern blot analysis, and leading to potential misclassification of the MLL gene status in leukaemia patients.

\section{Patients and methods}

Between October 1996 and April 1997, pretreatment bone marrow samples were obtained from six paediatric patients (RUPN (refers to unique patient number) $89,91,92,96,97$, and 99: four B cell precursor ALLs and two T cell ALLs) admitted to Roswell Park Cancer Institute (RPCI) or the Children's Hospital of Buffalo. After informed consent for research studies was obtained from the patient's parents, $2.0 \mathrm{ml}$ of bone marrow was aspirated in unheparinised syringes, and transferred to standard $10 \mathrm{ml}$ plasma tubes (Becton Dickinson Labware, Franklin Lakes, New Jersey, USA) containing 143 USP units of sodium heparin. One third of each sample was processed immediately for DNA isolation. The remaining two thirds were incubated at room temperature $\left(19^{\circ} \mathrm{C}\right.$ ) for 24 hours (all six samples) and 48 hours (three samples) or 60 hours (three samples), respectively, before genomic DNA was isolated using standard procedures. ${ }^{17}$ Briefly, after separation of mononuclear cells from the bone marrow sample by Ficoll-Hypaque (Sigma, St Louis, Missouri, USA) density centrifugation, DNA was purified by a previously described salting out procedure, ${ }^{17}$ and analysed for MLL gene rearrangements by Southern blot analysis. Aliquots of $10 \mu \mathrm{g}$ of genomic DNA were subjected to restriction enzyme digestion with either BamHI, SstI, or HindIII (Life Technologies, Grand Island, New York, USA), separated by electrophoresis on $0.8 \%$ agarose gels, transferred on to nitrocellulose (Schleicher and Schuell, Keene, New Hampshire, USA), and immobilised by UV crosslinking. Southern blots were hybridised to a ${ }^{32} \mathrm{P}$ radiolabelled $0.7 \mathrm{~kb}$ MLL cDNA fragment encompassing exons $5-7$ and $9-11$ (probe $0.7 \mathrm{~B}$ from reference 21) at $42^{\circ} \mathrm{C}$. Final washing conditions were $0.1 \% \mathrm{SDS} / 0.1 \times \mathrm{SSC}(1 \times \mathrm{SSC}=0.15 \mathrm{M} \mathrm{NaCl}$, $0.015 \mathrm{M}$ sodium citrate) at $52^{\circ} \mathrm{C}$. Autoradiography of blots was performed for three days at $-70^{\circ} \mathrm{C}$ with an intensifying screen.

In addition, genomic DNA was isolated from cryopreserved leukaemia samples obtained from 70 paediatric patients (RUPN 1-70; 54 ALLs, 16 AMLs) evaluated at RPCI between 1987 and 1994. This DNA was digested with BamHI, size fractionated on $0.8 \%$ agarose gels, Southern blotted, and hybridised to the $0.7 \mathrm{~B}$ MLL cDNA probe as described above. 


\section{Results}

We detected clear evidence of an MLL pseudo-rearrangement in two patients (RUPN 96 and 97) after their bone marrow was incubated ex vivo at room temperature for 48 or 60 hours, respectively. The patient characteristics are given in table 1 . Figure 1 displays a map of the MLL breakpoint cluster region. Figure 2A shows the Southern blot hybridisation pattern obtained in patient RUPN 96 using the 0.7B MLL cDNA probe. While only the germline $8.3 \mathrm{~kb}$ fragment is detected in genomic DNA isolated immediately after the sample was obtained, after 60

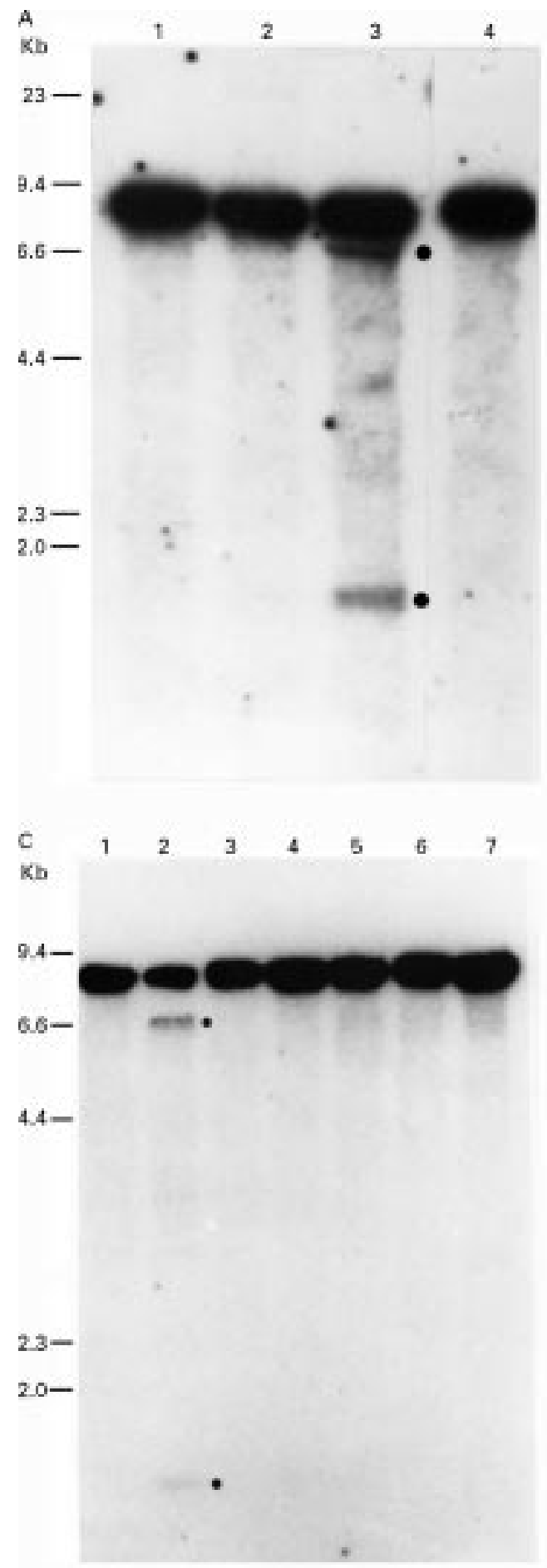

hours ex vivo incubation at room temperature, two additional, non-germline fragments of $6.8 \mathrm{~kb}$ and $1.5 \mathrm{~kb}$ are identified. These discrete, non-germline bands can easily be misinterpreted as rearranged fragments representing an MLL translocation breakpoint. Figure 2B shows DNA from patient RUPN 97 whose bone marrow was incubated for 24 and 48 hours at room temperature. The 48 hour time point clearly showed a pseudorearrangement of the MLL gene as well. Different digests confirmed that the MLL breakpoint cluster region is disrupted at the same unique site previously described as the

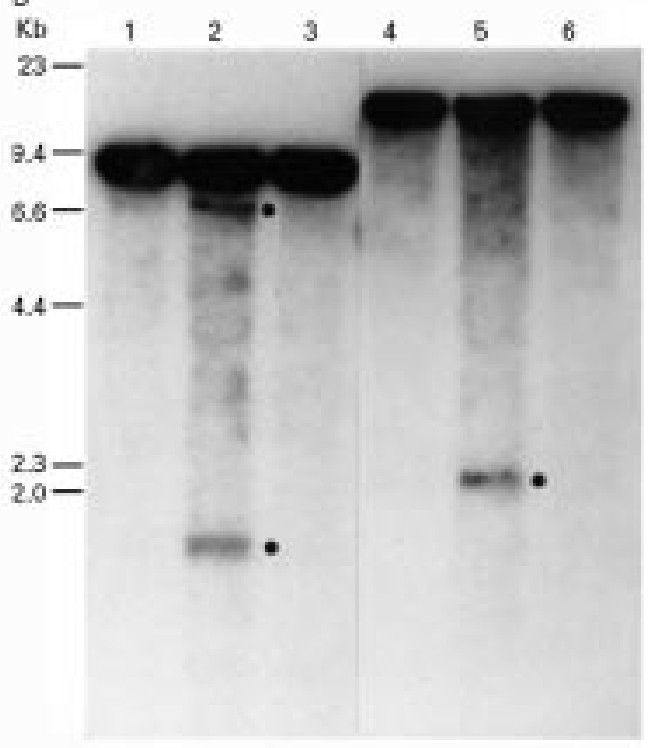

Figure 2 (A) Southern blot hybridisation of BamHI digested genomic DNA of patient RUPN 96 to the $0.7 B$ $M L L$ cDNA probe. DNA was isolated from the patient's bone marrow immediately after the sample was obtained (lane 1), after 24 hours (lane 2), and after 60 hours (lane 3). Genomic DNA from CEM, a cell line known to have a germline MLL configuration, was used as a control (lane 4). Two bands (indicated by closed circles) in addition to the $8.3 \mathrm{~kb}$ germline band are clearly seen at $6.8 \mathrm{~kb}$ and $1.5 \mathrm{~kb}$ in lane 3. Size standards are in kb. (B) Southern blot hybridisation of genomic DNA isolated from bone marrow of patient RUPN 97 immediately after the sample was obtained (lanes 1 and 4) and after 48 hours ex vivo incubation (lanes 2 and 5). Two different restriction enzyme digests are shown (BamHI, lanes 1-3; and HindIII, lanes 4-6). Additional bands induced by ex vivo incubation are visible in the 48 hour lanes and are indicated by closed circles. Genomic DNA from the CEM cell line was used as a control (lanes 3 and 6). Size standards are in $k b$. (C) Southern blot hybridisation to the $0.7 B M L L c D N A$ probe of BamHI digested genomic DNA obtained from cryopreserved leukaemic specimens. In patient RUPN 42 (lane 2), two additional bands of $6.8 \mathrm{~kb}$ and $1.5 \mathrm{~kb}$ are visible (indicated by closed circles). The control lane (lane 7) contains BamHI digested DNA from the U937 cell line, known to have a germline MLL configuration. Size standards are in $k b$. 


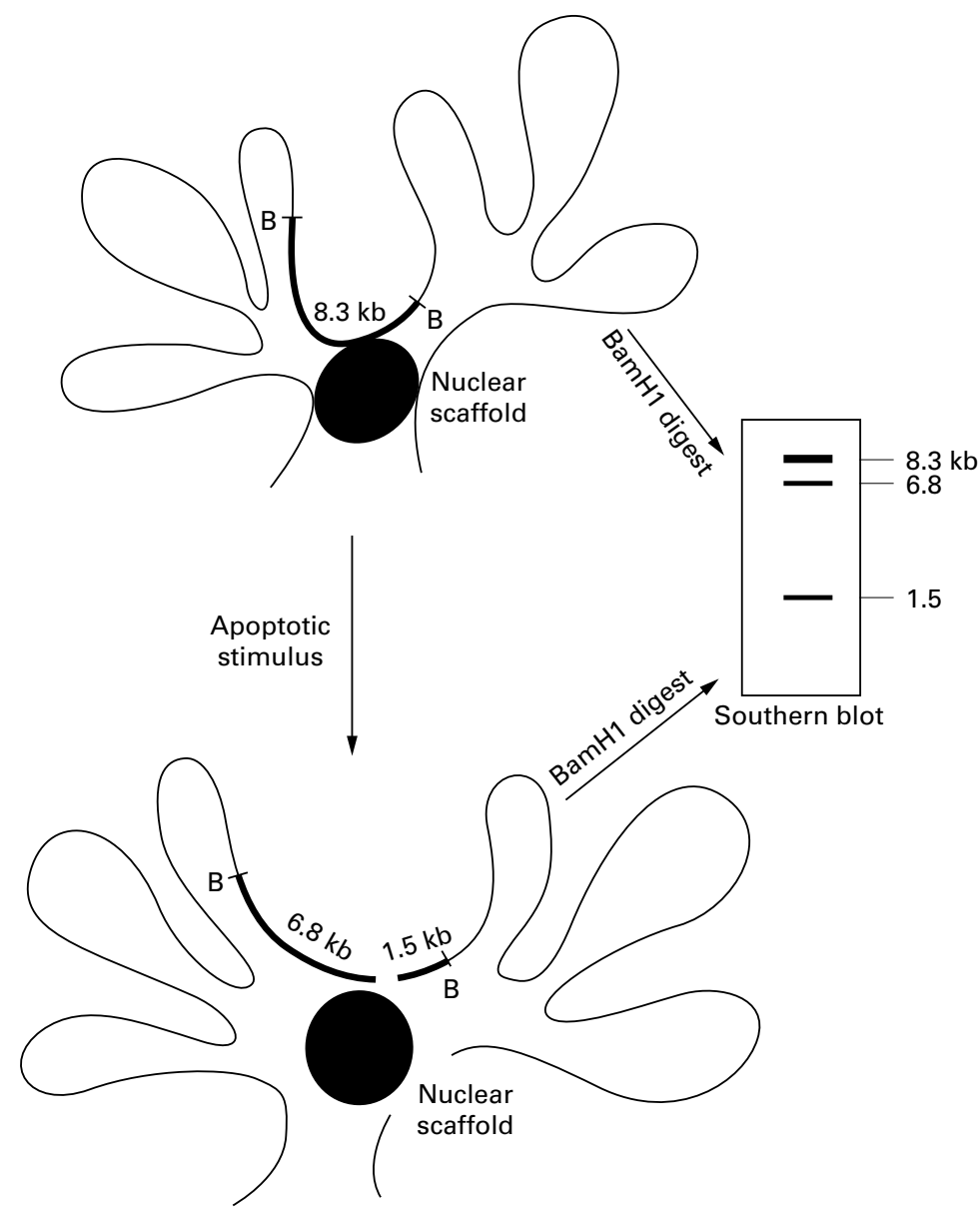

Figure 3 Model for the generation of an MLL pseudo-rearrangement by the higher order chromatin fragmentation associated with apoptosis. Discrete regions of genomic DNA, including a portion of the MLL breakpoint cluster region, ${ }^{26}$ are attached to the nuclear scaffold (upper panel). In response to an apoptotic stimulus, genomic DNA is initially cleaved into large (50-300 kb) fragments. ${ }^{24}$ One of the genomic regions cleaved by this process is the scaffold attachment region within the MLL breakpoint cluster region (lower panel). When genomic DNA is extracted from a cell population containing cells undergoing apoptosis and digested with BamHI, two fragments of $6.8 \mathrm{~kb}$ and $1.5 \mathrm{~kb}$ are generated, in addition to the $8.3 \mathrm{~kb}$ germline fragment. One end of both the $6.8 \mathrm{~kb}$ and $1.5 \mathrm{~kb}$ fragments is produced by the apoptosis induced cleavage, the other is produced by the BamHI restriction enzyme. $B$, BamHI restriction site.

target for apoptotic higher order chromatin fragmentation $^{18}$ (see map in fig 1 ; additional SstI digest not shown).

From October 1996 to April 1997, four additional diagnostic bone marrow samples (two B cell precursor ALLs, two T cell ALLs) were screened for MLL pseudorearrangements after 24 hours (all patients) and 48 hours (two patients) or 60 hours (two patients) incubation at room temperature. Two patients showed no evidence of an MLL pseudo-rearrangement, while two patients (one B cell precursor ALL after 60 hours; one T cell ALL after 48 hours) showed equivocal evidence of a pseudo-rearrangement. These patients could not be definitively scored as positive owing to high background hybridisation signals (data not shown).

We also screened cryopreserved leukaemic cells obtained at diagnosis from 70 paediatric patients with acute leukaemia evaluated at RPCI. One of these patients (RUPN 42), a 10 year old white girl with B cell ALL and L3 morphology, demonstrated an MLL pseudorearrangement (table 1; fig 2C).

\section{Discussion}

In the present study, we report the ex vivo generation of an MLL pseudo-rearrangement in leukaemic bone marrow samples, most likely caused by the higher order chromatin fragmentation that occurs during the early stages of apoptosis. To understand the mechanism which underlies these pseudo-rearrangements, it is important to visualise the MLL locus in its normal chromosomal context. Eukaryotic genomic DNA is organised in loops or groupings of loops that are periodically attached to a structure termed the nuclear scaffold. ${ }^{22}{ }^{23}$ This nuclear scaffold is a skeleton-like proteinaceous component with structural and functional properties in the nucleus. During the initial stages of apoptosis, nuclear DNA is cleaved into large fragments of $50-300 \mathrm{~kb}$. It has been suggested that these fragments represent DNA loops cleaved at regions where the DNA is attached to the nuclear scaffold ${ }^{24} 25$ (scaffold attachment regions). Figure 3 presents a model of higher order chromatin fragmentation during the early stages of apoptosis in context with the MLL breakpoint cluster region. Here, consistent with the above described higher order chromatin fragmentation model, specific cleavage occurs at a unique site, ${ }^{18}$ which maps to a previously identified scaffold attachment region within the MLL breakpoint cluster region. ${ }^{26}$ This site specific cleavage leads to the production of nongermline sized MLL fragments when digested with restriction enzymes, generating a pseudorearrangement of the MLL gene in Southern blot analysis. This process has important implications for the accuracy of MLL genotype analysis and may have an impact on genotype analysis by Southern blot at other loci as well. Conceptually, these pseudo-rearrangements may lead to misinterpretation of any Southern blot assay that depends on identification of non-germline fragments, such as triplet repeat expansions or restriction fragment length polymorphism (RFLP) analysis.

Cytogenetic and molecular genetic analyses are important variables in risk assessment strategies of patients with haematological malignancies, and are used with increasing frequency for therapeutic stratification of such patients. ${ }^{6728}$ The MLL gene at chromosome 11q23 is frequently disrupted in haematological malignancies, and MLL gene rearrangements have been shown to confer a poor prognosis in patients with ALL. ${ }^{6131416}$ Therefore, it is clear that proper classification of a leukaemic patient's MLL gene status is an important issue, because patients with MLL gene rearrangements may benefit from more intensive therapy, including allogenic bone marrow transplantation. ${ }^{5-7}$ Thus, recognition of an MLL pseudo-rearrangement has important clinical implications because this recognition may avoid misclassification of patients into high risk strata, and as a consequence prevent their exposure to unnecessary hazards associated with the intensive therapy given to patients with genuine MLL gene rearrangements. Furthermore, the recognition of such MLL pseudo-rearrangements will contribute to a less 
biased evaluation of prognostic values of ALL gene rearrangements in clinical studies, and help to avoid misclassification bias in ongoing epidemiological studies of the aetiology of leukaemias with 11q23/MLL rearrangements.

Site specific MLL cleavage leading to the described MLL pseudo-rearrangement is not limited to ALL. While our preliminary series includes B cell precursor ALL and B cell ALL patients, recently, Macintyre et al reported MLL gene rearrangements identical to the MLL pseudo-rearrangements described here in $\sim 5 \%$ of de novo AML patients of diverse French-American-British classification. ${ }^{29}$

It is unclear whether pretreatment MLL pseudo-rearrangements can occur in vivo, particularly in patients with a disease phenotype susceptible to apoptosis. Obviously, if these pseudo-rearrangements occur in vivo, even optimally processed samples may contain pseudo-rearrangements. In this regard, it is notable that in our single institution series, where leukaemic samples were generally processed and cryopreserved within four hours of sample procurement, the only patient whose cells demonstrated an MLL pseudorearrangement was a B cell ALL patient, a disease group highly susceptible to apoptosis. At present, the principal recommendations that can be made in order to avoid misclassification based on MLL pseudo-rearrangements are that samples be processed rapidly, and that the individuals interpreting these Southern blots should be aware of the restriction fragment pattern produced by MLL pseudorearrangements.

In conclusion, we report that generation of a pseudo-rearrangement of the MLL gene at chromosome 11q23 can be induced solely through ex vivo incubation of heparinised bone marrow samples for 24-60 hours at room temperature, simulating delays in sample processing that may occur during shipment to reference laboratories. We emphasise that the unique MLL fragmentation pattern shown in fig 1 does not represent an MLL translocation breakpoint, but rather a pseudo-rearrangement caused by apoptosis, and suggest that extreme caution should be used in the interpretation of any sample that demonstrates such a pseudorearrangement. Furthermore, although we have only demonstrated pseudorearrangements of the MLL gene, it is possible that other loci may also show pseudorearrangements, leading to misclassification and suboptimal therapy.

We would like to thank Ms P Pajak for artwork, and Mr D Chervinsky for technical assistance. This work was supported by grants from the NIH (CA73773-01 and CA16056-21), RPC Alliance (PDA), and the Deutsche Forschungsgemeinschaft
(MS). PDA is a scholar of the Leukemia Society of America.

1 Ziemin-van der Poel S, McCabe NR, Gill HJ, et al. Identification of a gene, MLL, that spans the breakpoint in $11 \mathrm{q} 23$ translocations associated with human leukemias. Proc Nat Acad Sci USA 1991;88:10735-9.
2 Djabali M, Selleri L, Parry P, et al. A trithorax-like gene is interrupted by chromosome 11 q 23 translocations in acute interrupted by chromosome 11q23 tran

3 Gu Y, Nakamura T, Alder H, et al. The t $(4 ; 11)$ chromosome translocation of human acute leukemias fuses the ALL-1 gene, related to Drosophila trithorax, to the AF-4 gene. Cell 1992;71:701-8 Tkachuk D, Kohler S, Cleary M. Involvement of a homolog
of Drosophila trithorax by 11q23 chromosomal translocations in acute leukemias. Cell 1992;71:691-700.

5 Thirman MJ, Gill HJ, Burnett RC, et al. Rearrangement of the MLL gene in acute lymphoblastic and acute myeloid leukemias with $11 \mathrm{q} 23$ chromosomal translocations. N Eng $7 \mathrm{Med}$ 1993;329:909-14.

6 Rubnitz JE, Behm FG, Downing JR. 11q23 rearrangements in acute leukemia. Leukemia 1996;10:74-82.

7 Hilden JM, Frestesdt JL, Kersey JH. Molecular analysis of Hilden JM, Frestesdt JL, Kersey JH. Molecular analysis
infant acute leukemia. Leuk Lymphoma 1997;25:191-9.

8 Gill-Super HJ, McCabe NR, Thirman MJ, et al. Rearrangements of the MLL gene in therapy-related acute myeloid leukemia in patients previously treated with agents targeting DNA topoisomerase II. Blood 1993;82:3705-11.

9 Bower M, Parry P, Gibbons B, et al. Human trithorax gene rearrangements in therapy-related acute leukemia after etoposide treatment. Leukemia 1994;8:226-9.

10 Cleary ML. A promiscuous oncogene in acute leukemia. $N$ Engl f Med 1993;329:958-9.

11 Smith M, Bleyer A, Crist W, et al. Uniform criteria for childhood acute lymphoblastic leukemia risk classification. $\mathcal{f}$ 1996;14:680-1.

12 Caligiuri MA, Strout MP, Gilliland DG. Molecular biology of acute myeloid leukemia. Semin Oncol 1997;24:32-44.

13 Chen C-S, Sorensen PHB, Domer PH, et al. Molecular rearrangements on chromosome $11 \mathrm{q} 23$ predominate in infant acute lymphoblastic leukemia and are associated with specific biologic variables and a poor outcome. Blood 1993;81:2386-93.

14 Pui C-H, Behm FG, Downing JR, et al. 11q23/MLL rearrangement confers a poor prognosis in infants with acute lymphoblastic leukemia. f Clin Oncol 1994;12:90915.

15 Sorensen PHB, Chen C-S, Smith FO, et al. Molecular rearrangements of the MLL gene are present in most cases of infant acute myeloid leukemia and are strongly correlated with monocytic or myelomonocytic phenotypes. f Clin with monocytic or myest 1994;93:429-37.

16 Taki T, Ida K, Bessho F, et al. Frequency and clinical significance of MLL gene rearrangements in infant acute leukemia. Leukemia 1996;10:1303-7.

17 Aplan PD, Chervinsky DS, Stanulla M, et al. Site-specific DNA cleavage within the MLL breakpoint cluster region induced by topoisomerase II inhibitors. Blood 1996;87: 2649-58.

18 Stanulla M, Wang J, Chervinsky DS, et al. DNA cleavage within the MLL breakpoint cluster region is a specific event which occurs as part of higher-order chromatin fragmentation during the initial stages of apoptosis. Mol Cell Biol 1997;17:4070-9.

19 Wyllie AH, Kerr JFR, Currie AR. Cell death: the significance of apoptosis. Int Rev Cytol 1980;68:251-306.

20 Fisher DE. Apoptosis in cancer therapy: crossing the Fisher DE. Apoptosis in cancer

$21 \mathrm{McCabe}$ NR, Burnett RC, Gill HJ, et al. Cloning of cDNAs of the MLL gene that detect DNA rearrangements and altered RNA transcripts in human leukemic cells with 11 223 translocations. Proc Natl Acad Sci USA 1992;89: $11794-9$.

22 Berezney R, Coffey DS. Identification of a nuclear protein matrix. Biochem Biophys Res Commun 1974;60:1410-19.

23 Mircovich J, Mirault ME, Laemmli UK. Organization of the higher-order chromatin loop: specific DNA attachment sites on nuclear scaffold. Cell 1984;39:23-32.

24 Oberhammer FA, Wilson JW, Dive C, et al. Apoptotic death in epithelial cells: cleavage of DNA to 300 and/or $50 \mathrm{~kb}$ fragments prior to or in absence of internucleosomal fragfragments prior to or in absence of intern
mentation. $E M B O \mathcal{F} 1993 ; 12: 3679-84$.

25 Bortner CD, Oldenburg NBE, Cidlowski JA. The role of DNA fragmentation in apoptosis. Trends Cell Biol 1995;5: 21-6.

26 Strissel-Broeker PL, Gill-Super H, Thirman MJ, et al. Distribution of $11 \mathrm{q} 23$ breakpoints within the MLL breakpoint cluster region in de novo acute myeloid leukemia and in treatment-related acute myeloid leukemia: correlation with scaffold attachment regions and topoisomerase II binding sites. Blood 1996;87:1912-22.

27 Raimondi SC. Current status of cytogenetic research in childhood acute lymphoblastic leukemia. Blood 1993;81: 2237-51.

28 Mrózek K, Heinonen K, de la Chapelle A, et al. Clinical significance of cytogenetics in acute myeloid leukemia. Semin Oncol 1997;24:17-31.

29 Macintyre E, Bourquelot P, Leboeuf D, et al. MLL cleavage occurs in approximately $5 \%$ of de novo acute myeloid leukemia, including in patients analyzed before treatment induction. Blood 1997;89:2224-6. 